\title{
Microarray analysis of circular RNA expression profiles associated with gemcitabine resistance in pancreatic cancer cells
}

\author{
CHAO XU, YUE YU and FEI DING \\ Department of Gastroenterology, The Affiliated Provincial Hospital of Anhui Medical University, \\ Hefei, Anhui 230001, P.R. China
}

Received November 16, 2017; Accepted May 8, 2018

DOI: $10.3892 / o r .2018 .6450$

\begin{abstract}
Pancreatic cancer (PC) is one of the most malignant tumors of the digestive system due to its rapid progression, metastasis and resistance to chemotherapy. Gemcitabine (GEM) chemotherapy is the first-choice treatment for advanced PC. However, the effect of GEM-based chemotherapy on PC is limited due to the development of chemoresistance, and the molecular mechanisms underlying this resistance have yet to be investigated. Circular RNAs (circRNAs), which can function as microRNA sponges, have been found to be involved in the development of several types of cancer. However, research on circRNAs in PC drug resistance is limited. In the present study, the GEM-resistant PC cell line, SW1990/GZ, was successfully established by treating parental SW1990 cells in vitro with increasing concentrations of GEM in culture medium intermittently for 10 months. By analyzing the expression profiles of circRNAs in microarray between SW1990/GZ and parental SW1990 cells, we identified 26 upregulated and 55 downregulated circRNAs (fold change $\geq 2$ and $\mathrm{P}<0.05$ ) among 12,866 detected circRNAs in SW1990/GZ compared with SW1990 cells. Furthermore, the changes in the expression of six representative circRNAs was validated by reverse transcription-quantitative PCR. In addition, Kyoto Encyclopedia of Genes and Genomes pathway analysis and Gene Ontology analysis were performed. These analyses revealed that the dysregulated circRNAs regulated several cancer-related pathways, such as the mitogen-activated protein kinase (MAPK) and mammalian target of rapamycin (mTOR) signaling pathways, and may be involved in the biological process of the regulation of chemoresistance, including nucleic acid metabolic process and cellular response to stress. The present study
\end{abstract}

Correspondence to: Dr Chao Xu, Department of Gastroenterology, The Affiliated Provincial Hospital of Anhui Medical University, 17 Lujiang Road, Luyang, Hefei, Anhui 230001, P.R. China

E-mail: xuchaoseu@126.com

Key words: pancreatic cancer, circular RNAs, non-coding RNA, microarray undertook a comprehensive expression analysis and revealed the functional profiles of differentially expressed circRNAs associated with GEM-resistance in PC, thereby indicating the possible participation of these dysregulated circRNAs in the development of chemoresistance and providing novel potential therapeutic targets for PC.

\section{Introduction}

Pancreatic cancer (PC) is one of the most lethal human malignancies, with an overall five-year survival rate of $<5 \%$ (1). The high mortality rate associated with PC can be largely attributed to its highly aggressive nature, wherein local invasion and remote metastasis may occur during the early stages of carcinogenesis (2). Thus, the majority of patients diagnosed with PC cannot undergo surgery and chemotherapy is thus the main treatment option. At present, gemcitabine (GEM) is the first-line drug used in the treatment of PC. However, its therapeutic efficacy is far from satisfactory due to the inherent chemoresistance of PC (3). A previous study revealed that only $23.8 \%$ of GEM-treated patients received therapeutic benefits in their early stages of treatment (4). However, the majority of these patients faced therapeutic failure due to the obtained chemoresistance against GEM. Thus, a better understanding of the molecular mechanisms underlying the development of GEM chemoresistance is necessary to develop novel-targeted therapies to 'flip the switch' from drug resistance to susceptibility in PC.

Recently, a set of non-coding RNAs (ncRNAs), including microRNAs (miRNAs or miRs) and long non-coding RNAs (lncRNAs), have been found to be involved in $\mathrm{PC}$ pathogenesis. Circular RNAs (circRNAs) are a special class of endogenously expressed non-coding RNAs, which are featured with a covalently closed loop structure without a $5^{\prime}$ to $3^{\prime}$ polarity and polyadenylated tail (5). circRNAs are highly conserved in mammals and are mainly expressed in a cell type-specific or developmental stage-specific manner, indicating their involvement in various physiological and pathological processes (6-8). Currently, studies have confirmed that circRNAs contain conserved miRNA binding sites and function as miRNA sponges to modulate the expression of target genes. To date, the dysregulation of circRNAs has been reported in a set 
of human diseases, particularly in cancer development and progression (9-16). A recent study demonstrated that clusters of circRNAs were aberrantly expressed in PC compared with normal samples (17); however, the specific roles of circRNAs in PC pathogenesis remain unknown.

In the present study, to explore the roles of circRNAs in the development of chemoresistance in PC, we first developed a GEM-resistant PC cell line (SW1990/GZ) by exposing SW1990 PC cells to gradient concentrations of GEM. We then performed microarray analysis of SW1990/GZ cells, along with their parental control cells. Our results demonstrated several differentially expressed circRNAs that may be involved in the transformation of the GEM resistance of PC and may provide potential molecular biomarkers or therapeutic targets for PC in the future.

\section{Materials and methods}

Establishment of GEM-resistant cell line, SW1990/GZ. The PC cell line, SW1990, was purchased from the Institute of Biochemistry and Cell Biology of the Chinese Academy of Sciences (Shanghai, China). The GEM-resistant cell line, SW1990/GZ, was established by repeated subcultures in the presence of stepwise increases in GEM concentrations (Tocris Bioscience, Ellisville, MO, USA) during the growth of SW1990 cells. First, SW1990 cells were cultured in RPMI-1640 (HyClone Laboratories/GE Healthcare, Chicago, IL, USA) containing $10 \%$ fetal bovine serum (Gibco/Thermo Fisher Scientific, Inc., Waltham, MA, USA) and various concentrations of GEM. Subsequently, cell death was observed, and the median lethal dose of SW1990 cells was set to $0.07 \mu \mathrm{g} / \mathrm{ml}$. Subsequently, the SW1990 cells were cultured in medium containing GEM at a concentration of $0.1 \mu \mathrm{g} / \mathrm{ml}$. Following incubation at $37^{\circ} \mathrm{C}$ for $48 \mathrm{~h}$, the culture and dead cells were replaced with fresh drug-free medium. The remaining cells could then grow and probably enter the logarithmic phase of cell growth. They were passaged twice and then re-cultured in medium containing GEM at a concentration of $0.1 \mu \mathrm{g} / \mathrm{ml}$. The medium was then replaced with culture medium containing GEM at a concentration of $0.4 \mu \mathrm{g} / \mathrm{ml}$, and the cells were cultured with the aforementioned cycle progress, according to four-fold increase in the drug concentration. Finally, the cells were cultured in a medium with a drug concentration of $400 \mu \mathrm{g} / \mathrm{ml}$. Therefore, the filter viable cells produced a stable resistance to high concentrations of GEM. After 10 months, we had successfully acquired a stable GEM-resistant cell line designated as SW1990/GZ.

Drug sensitivity assay. Cells $\left(3 \times 10^{3}\right.$ cells/well) were seeded in 96-well plates. After $12 \mathrm{~h}$, the cells were exposed to increasing concentrations of $\operatorname{GEM}(0,25,50,100,150,200,250,300,350$ and $400 \mu \mathrm{g} / \mathrm{ml}$ ) and incubated at $37^{\circ} \mathrm{C}$ for $72 \mathrm{~h}$ to determine the $\mathrm{IC}_{50}$ value using Cell Counting Kit-8 assay (CCK-8; Dojindo Molecular Technologies, Inc., Kumamoto, Japan) to evaluate the sensitivity to GEM.

RNA extraction, purification and array hybridization. Total RNA was extracted from three samples of SW1990/GZ and SW1990 cells using TRIzol reagent (Invitrogen, Carlsbad, CA, USA) and treated with Rnase R (Epicentre, Madison, WI, USA) to remove linear RNA. Subsequently, enriched circRNA samples were amplified and transcribed into fluorescent cRNA by a random priming method (Arraystar Super RNA Labeling kit; Arraystar, Inc., Rockville, MD, USA). The labeled cRNAs were purified by the RNeasy mini kit (Qiagen $\mathrm{GmbH}$, Hilden, Germany). The concentration and specific activity of the labeled cRNAs (pmol Cy3/ $\mu \mathrm{g}$ cRNA) were determined by NanoDrop ND-1000 (NanoDrop Technologies, Inc., Wilmington, DE, USA). Labeled cRNA (1 $\mu 1$ each) was fragmented by adding $5 \mu 1$ of $10 \mathrm{X}$ blocking agent and $1 \mu 1$ of $25 \mathrm{X}$ fragmentation buffer, and the mixture was then heated at $60^{\circ} \mathrm{C}$ for $30 \mathrm{~min}$. Subsequently, the labeled cRNA was diluted with $25 \mu 1$ of $2 \mathrm{X}$ hybridization buffer. Finally, the labeled cRNA was hybridized using Human 8x15 K circRNA Array (Arraystar). The hybridized arrays were washed, fixed and scanned using the Agilent DNA Microarray Scanner G2505C (Agilent Technologies, Inc., Santa Clara, CA, USA).

Microarray data analysis. Agilent Feature Extraction software (version 11.0.1.1; Agilent Technologies) was used to analyze scanned images for raw data extraction. Quintile normalization and subsequent data processing were performed using the $\mathrm{R}$ software package $\mathrm{R}$ version 3.1.2 (Agilent Technologies), and low intensity filtering was performed. In the comparison of the cricRNA profiles of two groups using Student's t-test, the differences with a fold change (FC) of $\geq 2$ and $\mathrm{P}<0.05$ were considered statistically significant.

Reverse transcription-quantitative PCR (RT-qPCR). Total RNA was extracted from the cell samples using TRIzol reagent (Invitrogen/Thermo Fisher Scientific, Inc., Waltham, MA, USA). Briefly, cDNA was synthesized from $1 \mu \mathrm{g}$ of total RNA with SuperScript III Reverse Transcriptase (Invitrogen Japan) samples. Quantitative PCR (qPCR) was performed using a SYBR PrimeScript RT-PCR kit (Takara, Kyoto, Japan) on a Rotor-Gene 6000 real-time genetic analyzer (Corbett Life Science, Mortlake, Australia). Primer sequences for candidate genes are listed in Table I. The PCR program was initiated for $10 \mathrm{~min}$ at $95^{\circ} \mathrm{C}$ before 40 thermal cycles, each at $10 \mathrm{sec}$ at $95^{\circ} \mathrm{C}$ and $1 \mathrm{~min}$ at $60^{\circ} \mathrm{C}$ to collect fluorescent signals. The relative quantification of circRNA expression levels was determined by taking the average of the GAPDH-normalized $2^{-\Delta \Delta C q}$ values (18).

GO and pathway analyses. The potential functions of the parental genes of differential circRNAs were analyzed using the Database for Annotation, Visualization and Integrated Discovery (DAVID; https://david.ncifcrf.gov/). The parental gene function was then predicted by GO functional annotation in terms of biological process (BP), cellular component (CC) and molecular function (MF). The results of the GO analysis are presented in a scatter plot, and the related pathways of the parental genes of differential circRNAs were analyzed by Kyoto Encyclopedia of Genes and Genomes (KEGG; http://www.genome.jp/kegg/).

miRNA prediction. The circRNA-miRNA interaction was predicted by using Arraystar home-made miRNA target prediction software (Arraystar), which is based on the TargetScan (http://www.targetscan.org/vert_72/) and 
Table I. The list of primers ( $\mathrm{F}$, forward and $\mathrm{R}$, reverse) used in this study.

\begin{tabular}{ll}
\hline circRNA & \multicolumn{1}{c}{ Primer sequence } \\
\hline circ_101543 & F: 5'-AAAAAGCACAGGCAGTTACTCA-3' \\
& R: 5'-CATTCCAGTAGGCGCTAAGA-3' \\
circ_000926 & F: 5'-TTGTGCTTTCTGGAGGGTCT-3' \\
& R: 5'-GCACAAATAAACCCCACATTTT-3' \\
circ_003251 & F: 5'-TATTATTCCCCCAGCTGCTC-3' \\
& R: 5'-CTGCTGCAACAGAAACCTGA-3' \\
circ_004077 & F: 5'-AAGATCCCGGATGACATGAG-3' \\
& R: 5'-GAGTCTTGGGAGGGTTGTCA-3' \\
circ_101672 & F: 5'-GGTTCTGCACCATCTTCAGG-3' \\
& R: 5'-TGGTGGTGGTCTTGTAGTCG-3' \\
circ_102747 & F: 5'-GTATCCTGGCCTGCCATC-3' \\
& R: 5'-TTGCCTCATCACCAACCA-3' \\
GAPDH & F:5'-CATGAGAAGTATGACAACAGCCT-3' \\
& R: 5'-AGTCCTTCCACGATACCAAAGT-3'
\end{tabular}

F, forward; R, reverse.

miRanda (http://www.microrna.org/) prediction algorithm. The differentially expressed circRNAs in all the comparisons were annotated in detail with the circRNA/miRNA interaction information.

CircRNA-miRNA-target gene network. To further elucidate the associations between circRNAs and miRNAs, potential circRNA-miRNA-target gene interaction analysis was conducted by Cytoscape (version 3.6.1; http://www.cytoscape. org/). The size of each node represents the number of putative miRNA functionally connected to each circRNA.

Statistical analysis. Statistical analysis was conducted using SPSS 13.0 software (SPSS, Inc., Chicago, IL, USA). The Student's t test was used for comparisons between two groups. $\mathrm{P}<0.05$ was considered to indicate a statistically significant difference.

\section{Results}

Drug resistance index of SW1990/GZ. The GEM resistance of the SW1990/GZ cell line was identified by determining its $\mathrm{IC}_{50}$ value against the parental SW1990 cell line. The $\mathrm{IC}_{50}$ value of GEM for the SW1990/GZ cells was $79.3 \pm 5.31 \mu \mathrm{g} / \mathrm{ml}$, which was 26.2-fold higher than that of the parental cell line, SW1990 $(3.03 \pm 0.27 \mu \mathrm{g} / \mathrm{ml})$, indicating that the drug resistance index of SW1990/GZ cells relative to the parental SW1990 cells was 26.2 (Fig. 1). The GEM-resistant cell line, SW1990/GZ, was thus successfully established.

Overview of circRNA profiles. The expression of human circRNAs was screened in the SW1990 and SW1990/GZ cell samples using a microarray platform. Hierarchical clustering

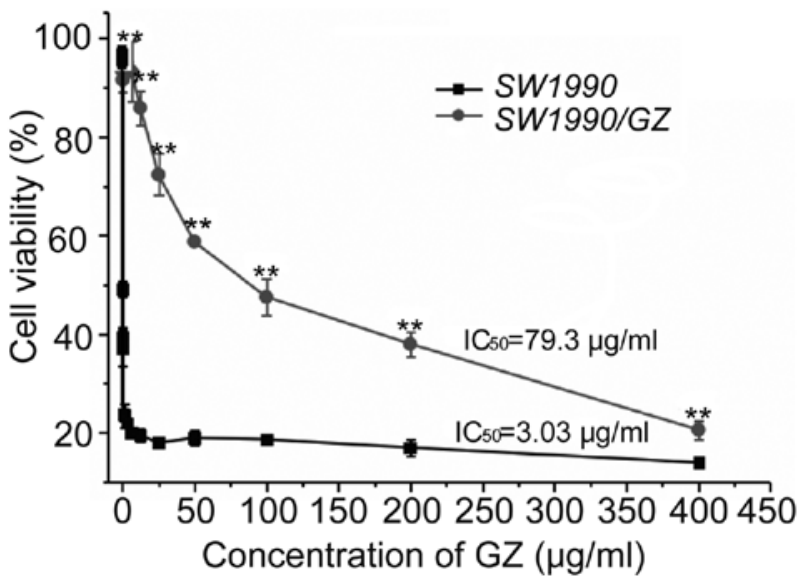

Figure 1. Cell viability of SW1990 and SW1990/GZ cells exposed to GEM. SW1990 and SW1990/GZ cells were treated with various concentrations of $\operatorname{GEM}(0,25,50,100,150,200,250,300,350$ and $400 \mu \mathrm{g} / 1)$ for $72 \mathrm{~h}$. Cell viability was determined by CCK-8 assay. Data shown are the mean \pm SEM. Each experiment was performed in triplicate. ${ }^{* *} \mathrm{P}<0.01$ vs. SW1990 cells. GEM, gemcitabine.

and box plot visualization revealed a distinguishable circRNA expression pattern between SW1990 and SW1990/GZ cells (Fig. 2A and B). In total, 81 circRNAs were differentially expressed (fold change $\geq 2.0$ and $\mathrm{P}<0.05$ ) between the SW1990/GZ and SW1990 cells. Among these, 26 circRNAs were upregulated and 55 circRNAs were downregulated $>2$-fold in SW1990/GZ cells. The 10 circRNAs with the most signficant increased and decreased in expression in the SW1990/GZ cells compared to the SW1990 cells are displayed in Fig. 2A and Table II. Significantly, the expression levels of circRNA_101672, circRNA_004077 and circRNA_003251 were upregulated in SW1990/GZ by 3.47-, 2.82- and 2.81-fold, respectively. Furthermore, circRNA_101543, circRNA_102747 and circRNA_000926 were downregulated by 3.94-, 3.88- and 3.51-fold, respectively. We also analyzed the chromosome distribution of these deregulated circRNAs. The differentially expressed circRNAs with statistically significance between SW1990/GZ and SW1990 cells were identified by volcano plot filtering (Fig. 2C). As displayed in Fig. 2D, each chromosome had circRNA locations, while chromosomes 1,2, 4, 5, 12 and 17 had considerably more circRNA locations than the other chromosomes, indicating a stronger association with GEM resistance in PC. The top two upregulated circRNAs, circRNA_101672 and circRNA_004077, were both located on chromosome 16, which may be the most important circRNA in GEM resistance in PC. Among the deregulated circRNAs, there were 72 exonic, 1 antisense, 3 intronic and 5 sense overlapping (Fig. 2E).

Validation of the microarray data by RT-qPCR. To validate the microarray data, three upregulated and three downregulated circRNAs were selected as representatives for further validation by RT-qPCR. According to the data shown in Fig. 3, four of the six tested circRNAs yielded results quite similar to those of the microarray. These well-validated circRNAs included two upregulated circRNAs (circRNA_101672 and circRNA_003251) and two downregulated circRNAs (circRNA_101543 and circRNA_102747). Although the other 

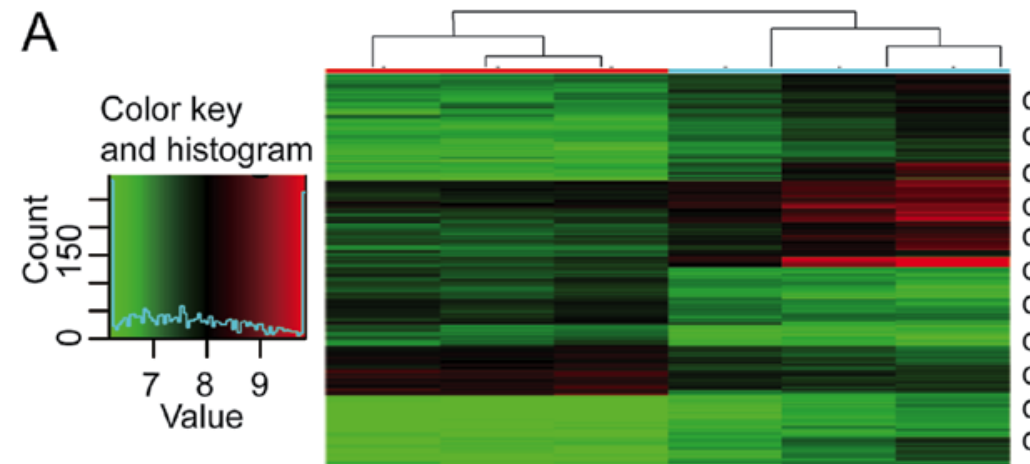

CircRNA 101672

circRNA_004077

CircRNA 003251

circRNA-102402

CircRNA-074298

circRNA-089762

circRNA 003596

circRNA-089761

circRNA 002178

circRNA-102403

circRNA 101543

circRNA 102747

circRNA_000926

circRNA 059665

circRNA-103827

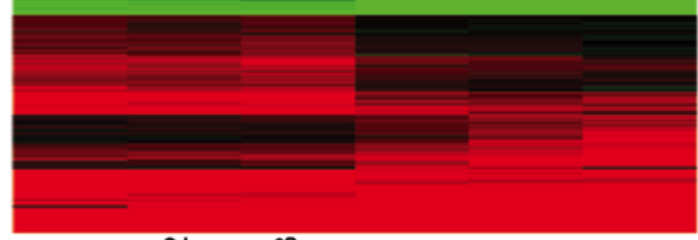

circRNA_406521

circRNA 103128

circRNA- 104490

circRNA-103829

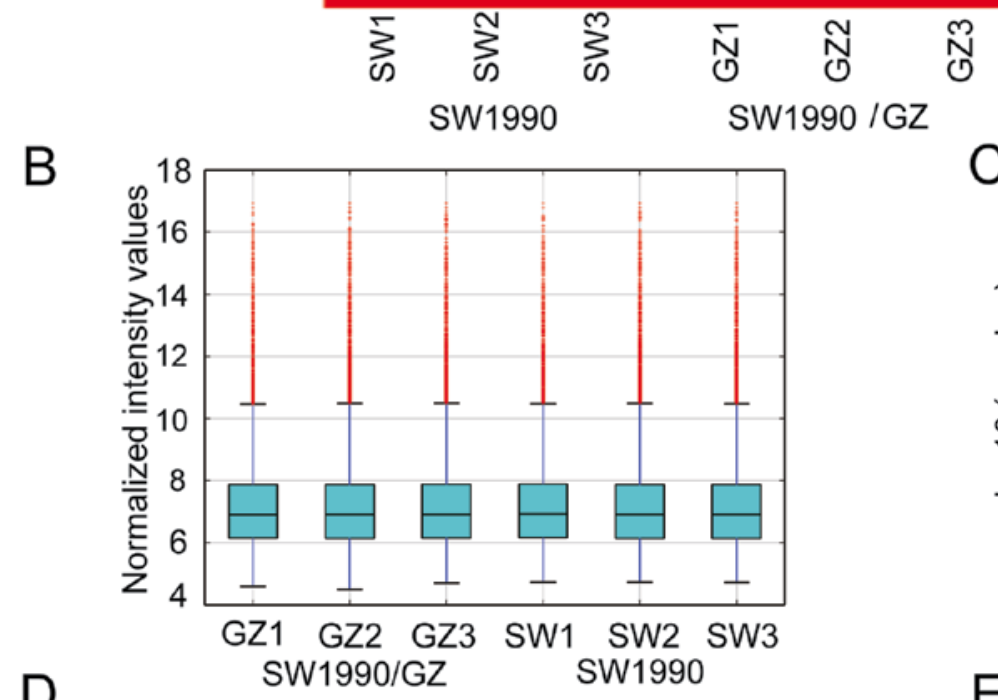

circRNA 070037

D

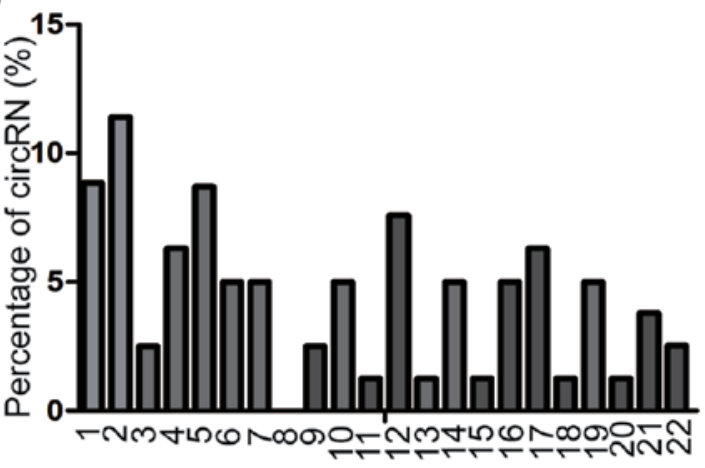

C

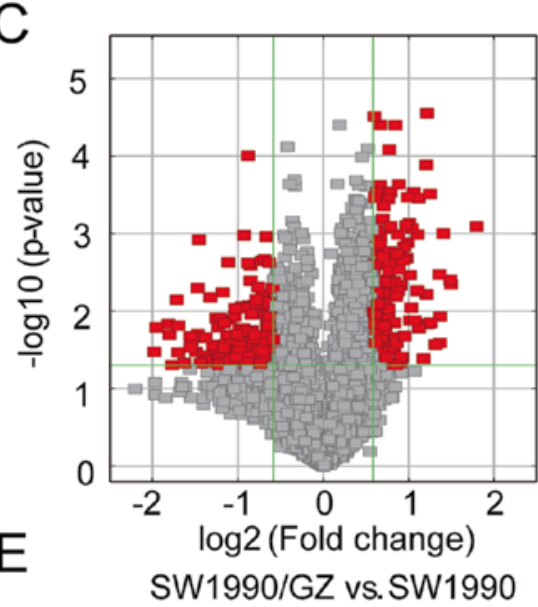

$1.20 \%$

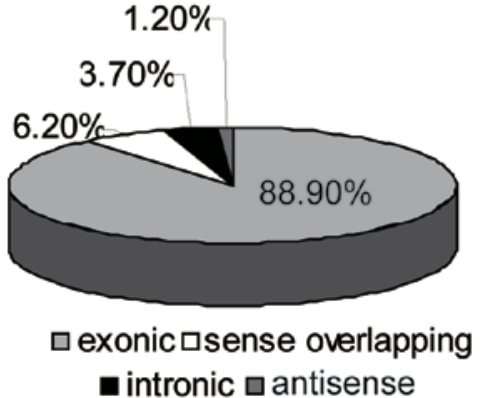

Figure 2. circRNA expression profile comparison between the SW1990/GZ and SW1990 cells. (A) Hierarchical clustering demonstrated a distinguishable circRNA expression profiling among SW1990/GZ and SW1990 cells. The top 10 most increased and decreased circRNAs in SW1990/GZ compared to SW1990 are shown. (B) Box plots demonstrating the distributions of a dataset for the circRNA profiles. After normalization, the distributions were nearly the same. (C) Volcano plot of the differentially expressed circRNAs. The $\mathrm{x}$ - and y-axes show the $\log 2$ fold change and negative $\log 10 \mathrm{P}$-value, respectively. The red points in the plot represent the differentially expressed circRNAs with statistical significance. (D) Chromosomal locations of differentially expressed circRNAs. The $\mathrm{x}$-axis denotes the ordinal of the chromosome, and the $\mathrm{y}$-axis denotes the percentage of circRNAs that were expressed differently between SW1990/GZ and parental SW1990 cells (fold change >2) (E). Types of variably expressed circRNAs based on their genomic proximity to the neighboring gene.

two circRNAs were not well repeated, the direction of change was similar to that noted in the microarray data. This result indicated that most of the circRNAs identified by microarray were reliable.
GO and pathway analysis of the parental genes of circRNAs. Differentially regulated circRNAs and their parental genes were further analyzed by GO analysis to speculate circRNA potential functions on three different aspects: BP, CC and 
Table II. Top dysregulated circRNAs in GEM-resistant PC cells.

\begin{tabular}{|c|c|c|c|c|c|}
\hline circRNA & Gene symbol & Type & Chrom & P-value & $\mathrm{FC}$ (abs) \\
\hline \multicolumn{6}{|c|}{ Upregulated circRNAs } \\
\hline circ_101672 & RAB40C & Exonic & chr16 & 0.000815496 & 3.4745157 \\
\hline circ_004077 & VAT1L & Exonic & chr16 & 0.003990298 & 2.8248131 \\
\hline circ_003251 & WNK1 & Exonic & $\operatorname{chr} 12$ & 0.004520208 & 2.8156161 \\
\hline circ_102402 & DAZAP1 & Exonic & chr19 & 0.000995122 & 2.646115 \\
\hline circ_074298 & HARS & Exonic & chr5 & 0.011756552 & 2.5846195 \\
\hline circ_089762 & JA760602 & Exonic & chrM & 0.025705535 & 2.5812949 \\
\hline circ_003596 & COL5A1 & Exonic & $\operatorname{chr} 9$ & 0.003354102 & 2.5655559 \\
\hline circ_089761 & JA760602 & Exonic & chrM & 0.027054021 & 2.4890659 \\
\hline circ_002178 & RPPH1 & Sense overlapping & chr14 & 0.014113033 & 2.3939466 \\
\hline circ_102403 & DAZAP1 & Exonic & chr19 & 0.000309541 & 2.3735941 \\
\hline \multicolumn{6}{|c|}{ Downregulated circRNAs } \\
\hline circRNA_101543 & VPS13C & Exonic & $\operatorname{chr} 15$ & 0.033434975 & 3.9419461 \\
\hline circRNA_102747 & ACTR2 & Exonic & $\operatorname{chr} 2$ & 0.016163204 & 3.8810596 \\
\hline circRNA_000926 & ACTR2 & Sense overlapping & $\operatorname{chr} 2$ & 0.014583979 & 3.5123931 \\
\hline circRNA_059665 & ABHD12 & Exonic & $\operatorname{chr} 20$ & 0.020206272 & 3.4890827 \\
\hline circRNA_103827 & HMGCS1 & Exonic & $\operatorname{chr} 5$ & 0.049403727 & 3.4092977 \\
\hline circRNA_406521 & UGT8 & Sense overlapping & $\operatorname{chr} 4$ & 0.007149841 & 3.283491 \\
\hline circRNA_103128 & DYRK1A & Exonic & $\operatorname{chr} 21$ & 0.034078531 & 3.2488121 \\
\hline circRNA_104490 & MKLN1 & Exonic & $\operatorname{chr} 7$ & 0.015401059 & 3.2463403 \\
\hline circRNA_103829 & HMGCS1 & Exonic & $\operatorname{chr} 5$ & 0.046511499 & 3.0960301 \\
\hline circRNA_070037 & NUP54 & Exonic & $\operatorname{chr} 4$ & 0.021180458 & 2.938704 \\
\hline
\end{tabular}

GEM, gemcitabine; PC, pancreatic cancer.

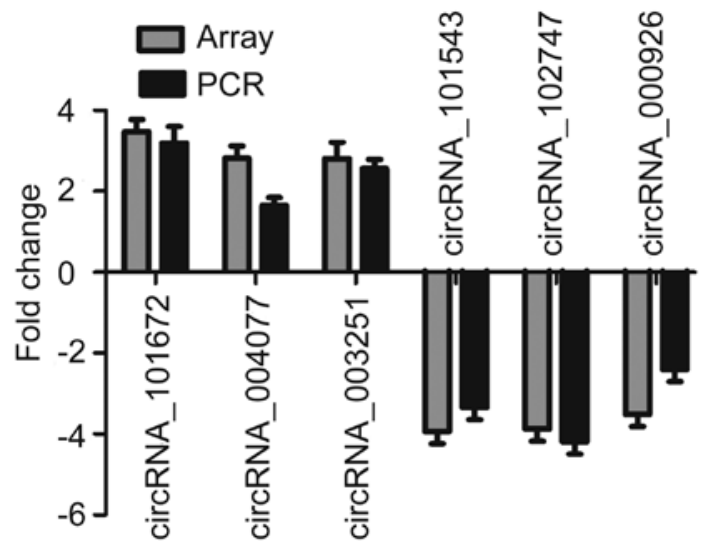

Figure 3. Validation of microarray data by RT-qPCR. Six differentially expressed circRNAs were validated by RT-qPCR. The y-axis column in the chart denotes the median fold changes.

MF. The top 10 enrichment GO entries for upregulated and downregulated circRNAs are displayed in Fig. 4. We found that the upregulated circRNAs were mainly enriched for GO terms related to nucleic acid metabolic process involved in BP, poly(A) RNA binding linked with MF, and nucleoplasm in CC. For the downregulated circRNAs, the most significantly enriched GO terms in BP, MF, and CC were peptidyl-threonine phosphorylation, enzyme binding and intracellular organelle, respectively. Furthermore, KEGG pathway analysis was performed. The upregulated circRNAs were involved in 11 pathways, while the downregulated circRNAs were involved in 37 pathways. The predominant pathways are displayed in Fig. 5. The top three enriched pathways for upregulated circRNAs were cell cycle, ubiquitin-mediated proteolysis and the cGMP-PKG signaling pathway. The top three enriched pathways for downregulated circRNAs were pancreatic cancer, EGFR tyrosine kinase inhibitor resistance and the mitogen-activated protein kinase (MAPK) signaling pathway. Among these enriched pathways, the MAPK and mammalian target of rapamycin (mTOR) signaling pathways have been previously reported to be involved in the chemoresistance of PC (19,20). As shown in Fig. 5C, the potential target genes of downregulated circRNAs incuding ATF2, BRAF, DUSP16, MAPK8, NFATC3, RAF1, TAB2, TAOK1 are directly associated with the MAPK signaling pathway.

Prediction for circRNA-miRNA interaction and circRNA-miRNA-target gene network. Given that circRNAs can function as sponges or inhibitors of their interacting miRNAs to regulate gene expression, circRNA-miRNA interaction was predicted with Arraystar homemade miRNA target prediction software based on TargetScan and miRanda. A total of 378 mature miRNAs were predicted to have docking sites in the differentially expressed circRNAs. Therefore, they can interact with the circRNAs. The circRNA-miRNA-target gene 


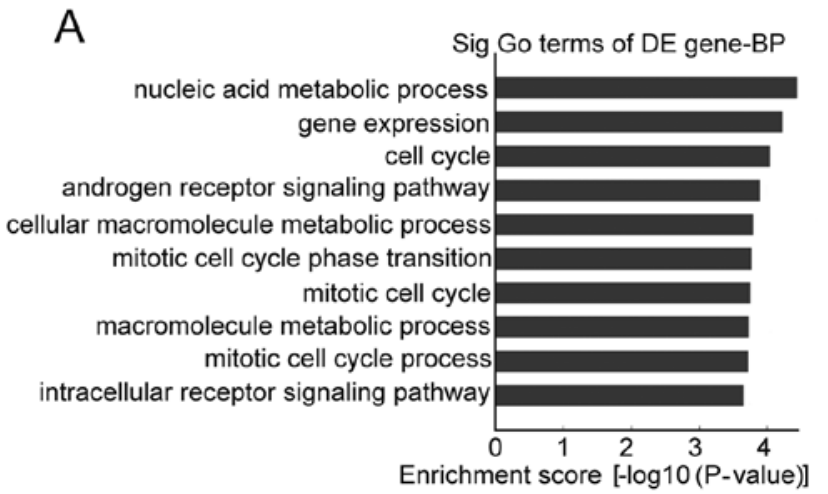

B
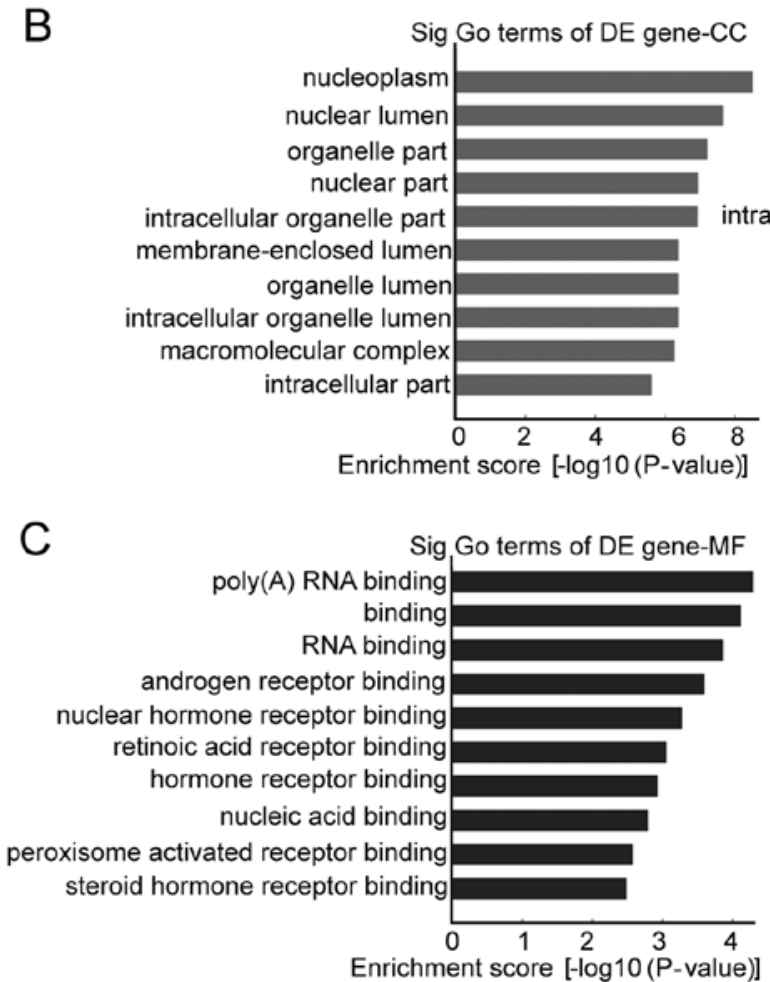

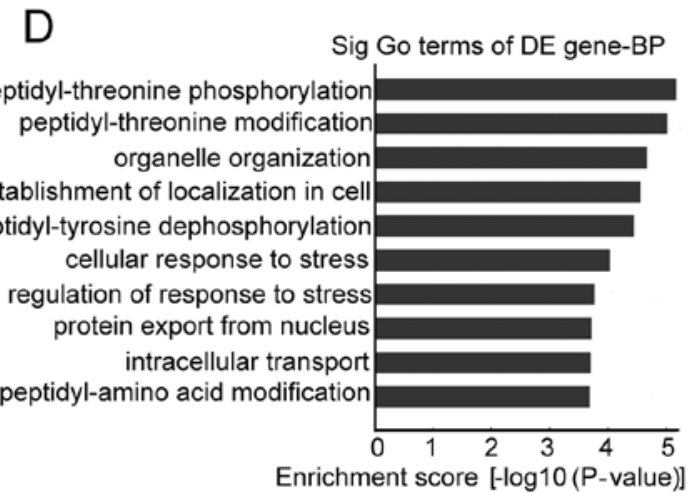

E

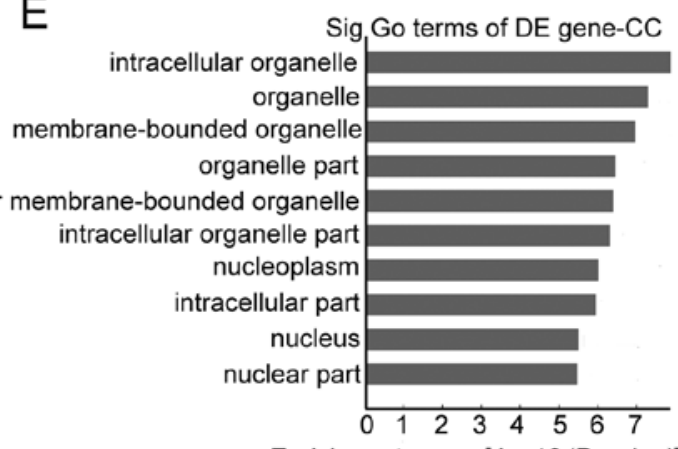

$\mathrm{F}$

Sig Go terms of DE gene-MF

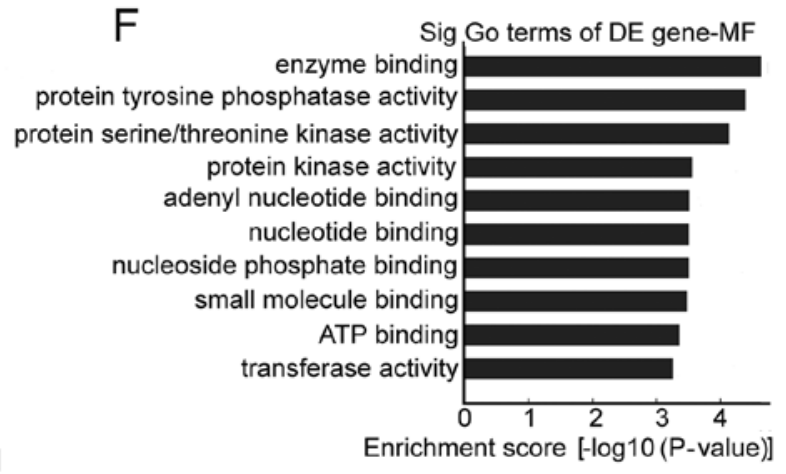
protein tyrosine phosphatase activity rotein serine/threonine kinase activity protein kinase activity phosphate binding Enrichment score $[$ - $\log 10$ (P-value)]

Figure 4. Gene ontology (GO) analysis of the parental genes of the dysregulated circRNAs. The GO analysis includes three categories: biological process (BP), cellular component (CC) and molecular function (MF). (A-C) GO enrichment corresponds to the upregulated circRNAs. (D-F) GO enrichment corresponds to the downregulated circRNAs. The P-value represents the significance of GO term enrichment in the dysregulated circRNA list. P-value $\leq 0.05$ was assigned to indicate the significance.

interacting network of the top 10 upregulated circRNAs was established (Fig. 6). circRNA_101672 was annotated in detail using the circRNA-miRNA interaction information (Fig. 7).

\section{Discussion}

GEM is the most efficient mono-drug therapy for treating PC. Thus, it is recommended as first-line therapeutic option for advanced PC patients. However, acquired GEM resistance contributes to treatment failure in a substantial number of PC patients. Thus, deciphering the mechanisms underlying GEM resistance is essential to overcome the problem. circRNAs were recently identified as novel functional endogenous ncRNAs, which can function as miRNA sponges, thereby interfering with the post-transcriptional actions of miRNAs as suppressors of the target genes (21). Accumulating evidence has indicated that circRNAs are involved in a number of human diseases, particularly in carcinomas, including hepatoma, neuroglioma, bladder carcinoma and breast cancer (22-25). Hence, circRNAs may serve as novel diagnostic and therapeutic strategies in human diseases. However, the changes in the expression of circRNAs and the related functional significance in PC chemoresistance has been rarely reported.

In the present study, we first generated a GEM-resistant PC cell line, SW1990/GZ, by stepwise selection and then analyzed the circRNA expression profiles between the SW1990/GZ and parental SW1990 cells with high-throughput circRNA microarrays to investigate the mechanisms of acquired GEM resistance. We observed that 26 circRNAs were upregulated, and 55 circRNAs were downregulated by $>2$-fold in the SW1990/GZ cells compared with the SW1990 cells. circRNA_101672, circRNA_004077 and circRNA_003251 were upregulated with top magnitudes. Conversely, circRNA_101543, circRNA_102747 and circRNA_000926 were downregulated with top magnitudes. The expression 
A

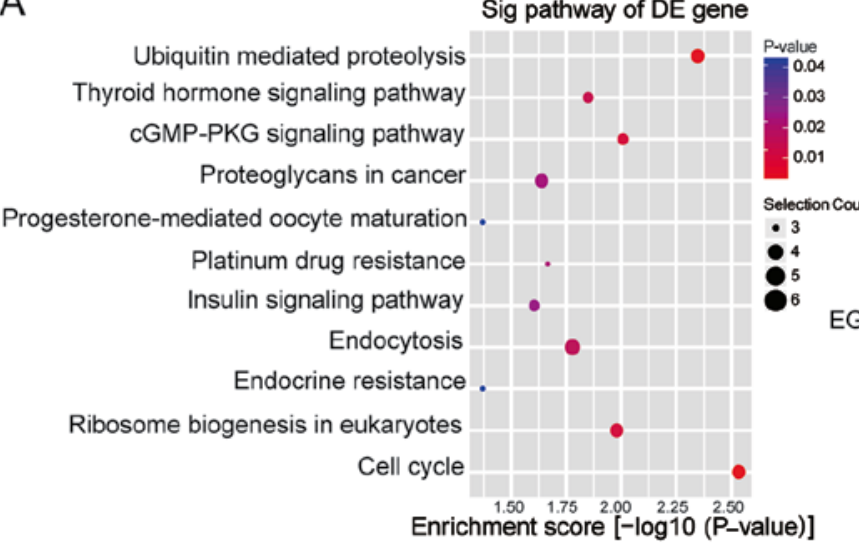

B

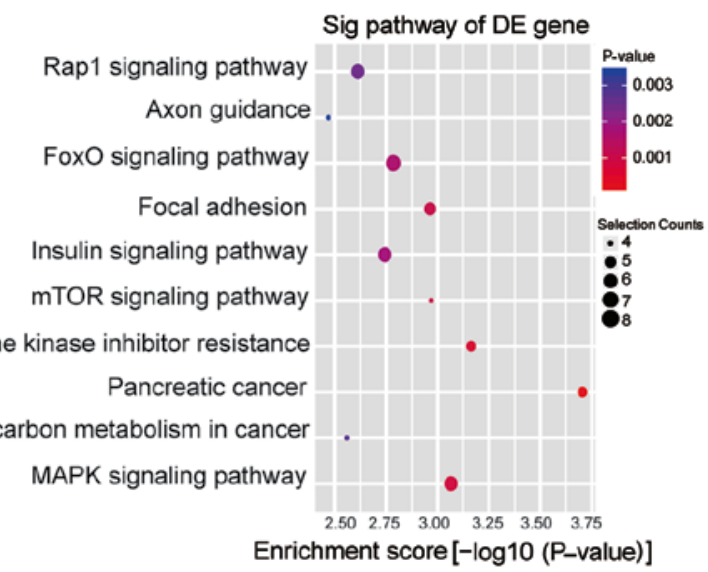

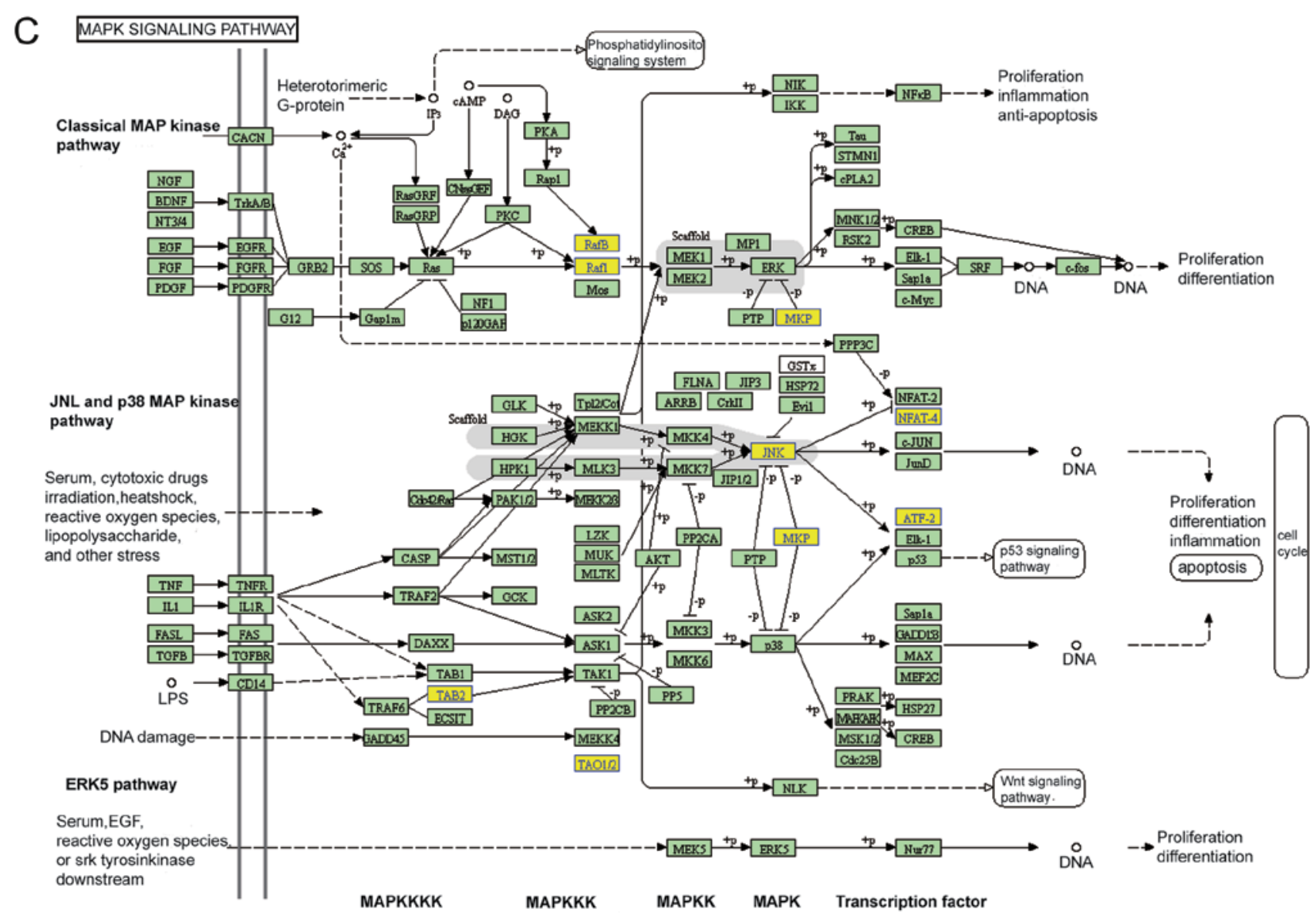

Figure 5. KEGG pathway analyses of the differential circRNA parental genes. The pathways correspond to (A) the upregulated circRNAs and (B) the downregulated circRNAs. The results are presented in a scatter plot. Enrichement factor refers to the ratio of the number of genes located in the pathway entry and the total number of genes in the pathway entry. (C) MAPK signaling pathway. Nodes marked in yellow are associated with target gene enrichment on MAPK signaling pathway.

patterns of the above-mentioned circRNAs were then validated by RT-qPCR, which revealed a high consistency between the RT-qPCR results and microarray data. Although we only used the SW1990 cell line as a cellular model in the present study, we hypothesize that some of the deregulated circRNAs in SW1990/GZ cells compared to SW1990 cells may be important and common contributors in PC-acquired GEM resistance. Our next step will be to confirm the results in other PC cell lines in our future study.

circRNAs are primarily generated from exons or introns of their parental genes and involved in the parental gene expression regulation (26-28). Thus, we investigated the biological functions and potential mechanisms of circRNAs in GEM resistance based on the GO and KEGG pathway analyses. GO enrichment analysis revealed that deregulated circRNAs were involved in the regulation of some crucial biological processes, such as cellular response to stress and cell cycle, which were important during the development of chemoresistance. Markedly, among these enriched pathways, the MAPK and mTOR signaling pathways have been shown to contribute to GEM chemoresistance $(29,30)$. Thus, some circRNAs may be involved in the GEM resistance of PC by regulating the above-mentioned signaling pathways. 


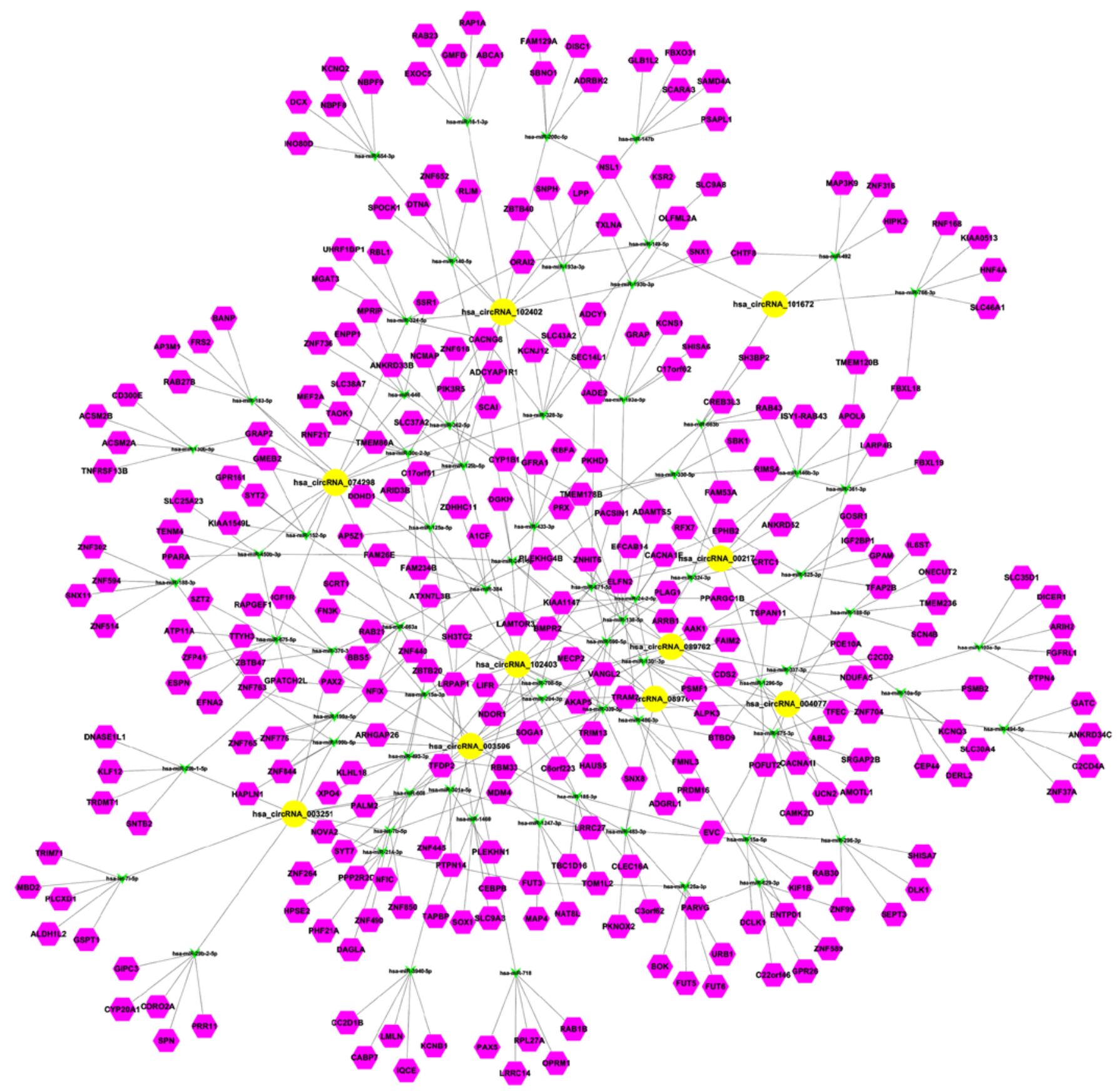

Figure 6. CircRNA-miRNA-target gene network analysis. Interactions between the top 10 upregulated circRNAs (shown by yellow nodes) and miRNAs and those between miRNAs and target genes in cancer signaling are shown in the map. The top 10 upregulated circRNAs are marked as yellow nodes. The pink nodes indicate the potetial target genes of the top 10 upregulated circRNAs.

Increasing evidence has demonstrated that circRNAs can 'sponge up' miRNAs to promote the expression of miRNA target protein-coding genes $(31,32)$. Given the important roles of miRNAs in the pathogenesis of PC, we hypothesized that some circRNAs may contribute to the GEM resistance of PC by interacting with miRNAs. Therefore, in this study, we performed in silico analyses to predict miRNAs targeted by these dysregulated circRNAs. For example, the upregulated circRNA with the largest fold change, circRNA_101672, potentially binds miR-492. A previous study indicated that miR-492 was involved in colon cancer chemoresistance via regulating the expression of CD147 (33). In addition, one of the top downregulated circRNAs, circ_102747, potentially binds miR-21. Hwang et al (34) and Dong et al (35) provided experimental evidence for the role of miR-21 in PC GEM resistance through modulation of apoptosis by directly regulating Bcl-2 and PTEN expression. In order to confirm whether these circRNAs are involved in PC chemoresistance, future studies, which will include the overexpression and knockdown of circRNA, their interaction with their potential targeted miRNAs and their involvement in GEM resistance in clinical $\mathrm{PC}$ samples are required. 


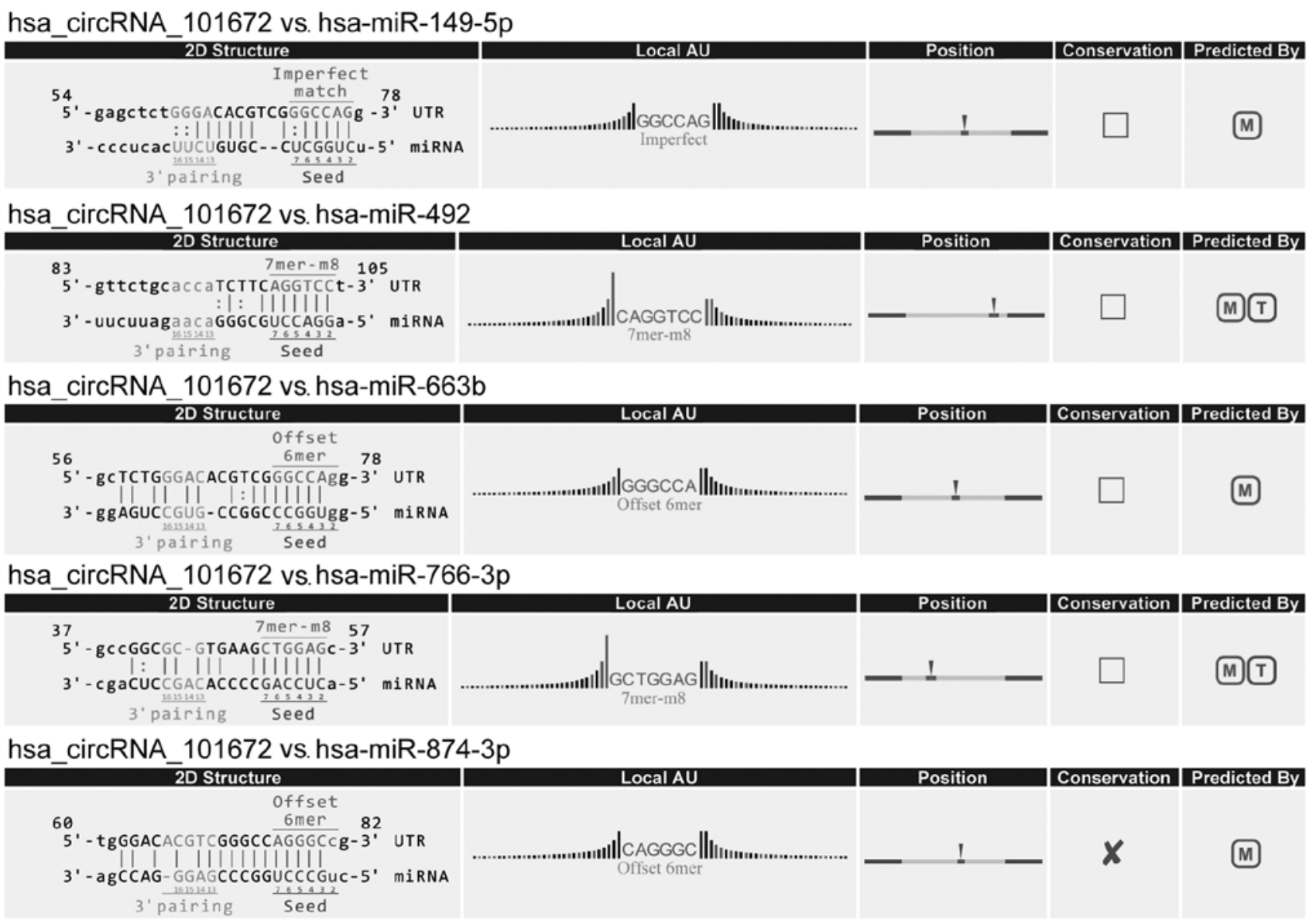

Figure 7. Detailed annotation for circRNA_101672-miRNA interaction. 7mer-m8: bases from number 2 to 8 match perfectly, and number 1 base is not A; offset 6mer: bases from number 3 to 8 match perfectly; imperfect match: imperfect base match was observed from number 2 to 7 . M: circRNA-miRNA interaction predicted by miRanda; T: circRNA-miRNA interaction predicted by TargetScan.

In conclusion, the present study revealed that circRNAs were dysregulated in the GEM-resistant PC cell line compared with its parental cell line. For these dysregulated circRNAs, we conducted GO enrichment and pathway analyses for their parental genes, which indicated that circRNAs may play important roles in the development of GEM resistance. By predicting the circRNA-miRNA interaction, we found several dysregulated circRNA, i.e., circ_101672 and circ_102747, which can potentially bind some miRNAs involved in cancer chemoresistance. These could be essential molecular mechanisms underlying the function of circRNAs in the chemoresistance of PC. Since our results were only based on an in vitro cell line model, clinical sample and in vivo validation is warranted in the future. On the whole, our findings revealed the potential roles of circRNAs in PC chemoresistance and potential therapeutic targets for circRNAs in PC treatment.

\section{Acknowledgements}

Not applicable.

\section{Funding}

This study was supported by the Natural Science Foundation of Anhui province (grant no. 1508085SQH224).

\section{Availability of data and materials}

The datasets used and/or analyzed during the current study are available from the corresponding author on reasonable request.

\section{Authors' contributions}

CX conceived and designed the study. CX and FD performed the research. CX wrote the manuscript. CX, YY and FD analyzed the data. All authors have read and approved the manuscript and agree to be accountable for all aspects of the research in ensuring that the accuracy or integrity of any part of the work are appropriately investigated and resolved.

\section{Ethics approval and consent to participate}

Not applicable.

\section{Consent for publication}

Not applicable.

\section{Competing interests}

The authors declare that they have no competing interests. 


\section{References}

1. Siegel R, Naishadham D and Jemal A: Cancer statistics, 2012 CA Cancer J Clin 62: 10-29, 2012

2. Yeo TP, Hruban RH, Leach SD, Wilentz RE, Sohn TA, Kern SE, Iacobuzio-Donahue CA, Maitra A, Goggins M, Canto MI, et al Pancreatic cancer. Curr Probl Cancer 26: 176-275, 2002.

3. Rosenberg L: Pancreatic cancer: A review of emerging therapies. Drugs 59: 1071-1089, 2000.

4. Mittal A, Chitkara D, Behrman SW and Mahato RI: Efficacy of gemcitabine conjugated and miR-NA-205 complexed micelles for treatment of advanced pancreatic cancer. Biomaterials 35: 7077-7087, 2014

5. Liu J, Liu T, Wang X and He A: Circles reshaping the RNA world: From waste to treasure. Mol Cancer 16: 58, 2017.

6. Salzman J: Circular RNA expression: Its potential regulation and function. Trends Genet 32: 309-316, 2016.

7. Bonizzato A, Gaffo E, Te Kronnie G and Bortoluzzi S: CircRNAs in hematopoiesis and hematological malignancies. Blood Cancer J 6: e483, 2016.

8. Li J, Yang J, Zhou P, Le Y, Zhou C, Wang S, Xu D, Lin H and Gong Z: Circular RNAs in cancer: Novel insights into origins, properties, functions and implications. Am J Cancer Res 5 : 472-480, 2015.

9. Taborda MI, Ramírez S and Bernal G: Circular RNAs in colorectal cancer: Possible roles in regulation of cancer cells. World J Gastrointest Oncol 9: 62-69, 2017.

10. Nair AA, Niu N, Tang X, Thompson KJ, Wang L, Kocher JP, Subramanian S and Kalari KR: Circular RNAs and their associations with breast cancer subtypes. Oncotarget 7: 80967-80979, 2016.

11. Yang P, Qiu Z, Jiang Y, Dong L, Yang W, Gu C, Li G and Zhu Y: Silencing of cZNF292 circular RNA suppresses human glioma tube formation via the $\mathrm{Wnt} / \beta$-catenin signaling pathway. Oncotarget 7: 63449-63455, 2016.

12. Huang M,Zhong Z,Lv M,Shu J,Tian Q and Chen J: Comprehensive analysis of differentially expressed profiles of lncRNAs and circRNAs with associated co-expression and ceRNA networks in bladder carcinoma. Oncotarget 7: 47186-47200, 2016.

13. Ahmed I, Karedath T, Andrews SS, Al-Azwani IK, Mohamoud YA, Querleu D, Rafii A and Malek JA: Altered expression pattern of circular RNAs in primary and metastatic sites of epithelial ovarian carcinoma. Oncotarget 7: 36366-36381, 2016.

14. Xie H, Ren X, Xin S, Lan X, Lu G, Lin Y, Yang S, Zeng Z, Liao W, Ding YQ and Liang L: Emerging roles of circRNA_001569 targeting miR-145 in the proliferation and invasion of colorectal cancer. Oncotarget 7: 26680-26691, 2016.

15. Fan X, Weng X, Zhao Y, Chen W, Gan T and Xu D: Circular RNAs in cardiovascular disease: An overview. Biomed Res Int 2017: 5135781, 2017

16. Van Rossum D, Verheijen BM and Pasterkamp RJ: Circular RNAs: Novel regulators of neuronal development. Front Mol Neurosci 9: 74, 2016.

17. Li HM, Hao XK, Wang HM, Liu ZC, He Y, Pu M, Zhang HT, Yu HC, Duan JL and Qu SB: Circular RNA expression profile of pancreatic ductal adenocarcinoma revealed by microarray. Cell Physiol Biochem 40: 1334-1344, 2016.

18. Livak KJ and Schmittgen TD: Analysis of relative gene expression data using real-time quantitative PCR and the $2^{-\Delta \Delta C_{\mathrm{T}}}$ method. Methods 25: 402-408, 2001.

19. Jin X, Pan YQ, Wang LG, Ma T, Zhang LZ, Tang AH, Billadeau DD, Wu HS and Huang HJ: Fructose-1,6-bisphosphatase Inhibits ERK activation and bypasses gemcitabine resistance in pancreatic cancer by blocking IQGAP1-MAPK interaction. Cancer Res 77: 4328-4341, 2017.
20. Kagawa S, Takano S, Yoshitomi H,Kimura F, Satoh M, Shimizu H, Yoshidome H, Ohtsuka M, Kato A, Furukawa K, et al: Akt/mTOR signaling pathway is crucial for gemcitabine resistance induced by Annexin II in pancreatic cancer cell. J Surg Res 178: 758-767, 2012.

21. Hansen TB, Jensen TI, Clausen BH, Bramsen JB, Finsen B, Damgaard CK and Kjems J: Natural RNA circles function as efficient microRNA sponges. Nature 495: 384-388, 2013.

22. Qin M, Liu G, Huo X, Tao X, Sun X, Ge Z, Yang J, Fan J, Liu L and Qin W: Hsa_circ_0001649: A circular RNA and potential novel biomarker for hepatocellular carcinoma. Cancer Biomark 16: 161-169, 2016.

23. Yang P, Qiu Z, Jiang Y, Dong L, Yang WS, Gu C, Li G and Zhu Y: Silencing of cZNF292 circular RNA suppresses human glioma tube formation via the Wnt/ $\beta$-catenin signaling pathway. Oncotarget 7: 63449-63455, 2016.

24. Zhong Z, Lv M and Chen J: Screening differential circular RNA expression profiles reveals the regulatory role of circTCF25-miR-103a-3p/miR-107-CDK6 pathway in bladder carcinoma. Sci Rep 6: 30919, 2016.

25. Yang W, Du WW, Li X, Yee AJ and Yang BB: Foxo3 activity promoted by non-coding effects of circular RNA and Foxo3 pseudogene in the inhibition of tumor growth and angiogenesis. Oncogene 35: 3919-3931, 2016.

26. Li F, Zhang L, Li W, Deng J, Zheng J, An M, Lu J and Zhou Y: CircularRNA ITCH has inhibitory effect on ESCC by suppressing the Wnt/ $/$-catenin pathway. Oncotarget 6: 6001-6013, 2015.

27. Zhang Y, Zhang XO, Chen T, Xiang JF, Yin QF, Xing YH, Zhu S, Yang $L$ and Chen LL: Circular intronic long noncoding RNAs. Mol Cell 51: 792-806, 2013.

28. Li Z, Huang C, Bao C, Chen L, Lin M, Wang X, Zhong G, Yu B, $\mathrm{Hu}$ W, Dai L, et al: Exon-intron circular RNAs regulate transcription in the nucleus. Nat Struct Mol Biol 22: 256-264, 2015.

29. Sui X, Kong N, Ye L, Han W, Zhou J, Zhang Q, He C and Pan H: p38 and JNK MAPK pathways control the balance of apoptosis and autophagy in response to chemotherapeutic agents. Cancer Lett 344: 174-179, 2014.

30. Kagawa S, Takano S, Yoshitomi H, Kimura F, Satoh M, Shimizu H, Yoshidome H, Ohtsuka M, Kato A, Furukawa K, et al: Akt/mTOR signaling pathway is crucial for gemcitabine resistance induced by Annexin II in pancreatic cancer cells. J Surg Res 178: 758-767, 2012.

31. Wang K, Long B, Liu F, Wang JX, Liu CY, Zhao B, Zhou LY, Sun T, Wang M, Yu T, et al: A circular RNA protects the heart from pathological hypertrophy and heart failure by targeting miR-223. Eur Heart J 37: 2602-2611, 2016.

32. Zheng Q, Bao C, Guo W, Li S, Chen J, Chen B, Luo Y, Lyu D, Li Y, Shi G, et al: Circular RNA profiling reveals an abundant circHIPK3 that regulates cell growth by sponging multiple miRNAs. Nat Commun 7: 11215, 2016.

33. Peng L, Zhu H, Wang J, Sui H, Zhang H, Jin C, Li L, Xu T and Miao R: MiR-492 is functionally involved in Oxaliplatin resistance in colon cancer cells LS174T via its regulating the expression of CD147. Mol Cell Biochem 405: 73-79, 2015.

34. Hwang JH, Voortman J, Giovannetti E, Steinberg SM, Leon LG, Kim YT,Funel N,Park JK, Kim MA, Kang GH, et al: Identification of microRNA-21 as a biomarker for chemoresistance and clinical outcome following adjuvant therapy in resectable pancreatic cancer. PLoS One 5: e10630, 2010

35. Dong J, Zhao Y, Zhou L, Zhang TP and Chen G: Bcl-2 upregulation induced by miR-21 via a direct interaction is associated with apoptosis and chemoresistance in MIA PaCa-2 pancreatic cancer cells. Arch Med Res 42: 8-14, 2011. 